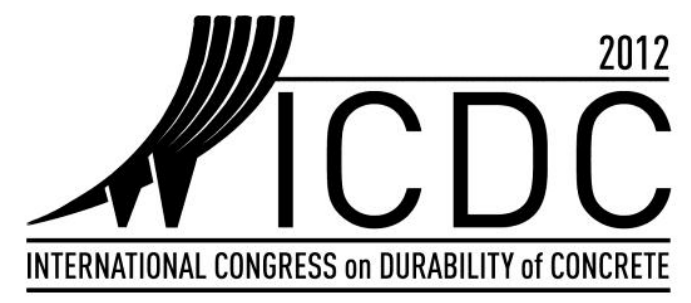

\title{
SERVICE LIFE AND LIFE CYCLE COST MODELLING OF CATHODIC PROTECTION SYSTEMS FOR CONCRETE STRUCTURES
}

\author{
Rob B. Polder (1,2), Greet Leegwater (1), Daniël Worm (1), Wim Courage (1) \\ (1) TNO Technical Sciences, P.O.Box 49, \\ 2600 AA Delft, The Netherlands, email rob.polder@tno.nl \\ (2) Delft University of Technology, Civil Engineering and Geosciences, \\ Delft, The Netherlands
}

\begin{abstract}
Corrosion of reinforcing steel in concrete structures causes concrete cracking, steel diameter reduction and eventually loss of safety. In infrastructure this is mainly due to penetration of chloride ions from de-icing or marine salts. Conventional repair means heavy, labour intensive and costly work and economic pressures (time and money) work against the required quality level. Consequently, conventional repair is short lived in many cases in practice. Corrosion reappears quickly and the structure needs to be repaired again after a relatively short time, further increasing life-cycle cost. A completely different situation applies to cathodic protection (CP) of steel in concrete as a repair method. Cathodic protection of reinforcing steel has been applied successfully to concrete structures with corrosion damage for more than 25 years.

This paper reports on past experience and presents a model for predicting life cycle costs for maintaining CP systems. Results will be presented from an inventory of CP systems in The Netherlands installed between 1987 and 2009 on about 150 structures, for which performance and maintenance data are reported. The large majority provides corrosion protection for a long time. Degradation of components and overall systems seems to occur in limited numbers. Failure of components and total systems as a function of age is quantified. On the average, the time until minor repairs of parts is necessary is about 15 years. Global failure of the anode, which necessitates near complete replacement of the system, is rare. Based on the statistical analysis of field data, the cost of maintaining a CP system is predicted using a life cycle cost model.
\end{abstract}

Key-words: service life; corrosion; cathodic protection; life cycle cost modeling; existing structures 


\section{INTRODUCTION}

Corrosion of reinforcing steel in concrete structures may occur, e.g. in bridges due to penetration of chloride ions from de-icing salts or sea water spray [1]. Reinforcement corrosion causes concrete cracking and steel diameter reduction, eventually resulting in loss of safety. Conventional repair means heavy, labour intensive and costly work. Economic pressures (time and money) work against the required quality level (perfect steel cleaning, removal of contaminated concrete). Consequently, conventional repair is short lived in many cases. Corrosion reappears quickly and structures need to be repaired again after a relatively short time, further increasing life-cycle cost. In a European study of the life of repairs (mainly patch repair), it was found that repairs had a short life in practice [2]. A completely different situation comes about with cathodic protection (CP) as a repair method. Cathodic protection of reinforcing steel has been applied to concrete structures with corrosion damage in Europe for about 25 years and slightly longer in the US [3, 4, 5, 6, 7]. A recent study reports on long term performance of CP systems in UK motorway structures [8].

Based on TNO's ambition to stimulate innovation and technical progress and its role as designer of CP systems for concrete structures, a research project was initiated, called CP+ Performance Toolbox. CP companies, concrete repair companies and consultants are participating in the project. It includes improved numerical modelling for design of CP systems and analysis of their service life, resulting in prototype tools for design and life cycle costing.

This paper presents results from a survey of CP systems in The Netherlands. Until 2009, about 150 structures have been provided with CP since 1987. Sufficient documentation of design, performance and maintenance is available for working life analysis of about 105 of those systems. The large majority provides corrosion protection for a long time. Degradation of components occurs in some systems and failure of components as a function of age has been quantified. In a limited number of cases, intervention (repair or replacement of parts) has been necessary for electrical connections, primary anodes, reference electrodes and power units. Failure of parts of the anode system has occurred with a rate that increases with age. Taking CP system life until interventions are necessary as the main criterion, the mean service life is about 15 years. However, complete failure of the anode was rare; and no cases have been reported where corrosion had reappeared.

Consequently, maintaining good corrosion protection in previously corroding concrete structures using CP over for example 25 years is mainly a matter of spending some money to maintain the $\mathrm{CP}$ system. Based on analysis of the field data a life cycle cost tool was built. Results will be given for example cases.

\section{COMPONENTS OF CP SYSTEMS}

A CP system for protection of steel in concrete basically consists of a conductor called the anode from which a small direct current flows through the concrete to the reinforcement, see Figure 1. The steel potential becomes more negative and corrosion is suppressed. The anode can be either directly applied to the concrete surface (e.g. a conductive coating) or consists of a mesh of activated titanium or carbon fibres embedded in a cementitious overlay. Other types comprise titanium strips in boreholes or slots filled with cementitious mortar. The anode material (with or without overlay) is fed by a primary anode (PA, a metal wire, mesh or strip), together they form 
the anode system. The anode system is linked through anode-copper connections and isolated copper cables to the power unit.

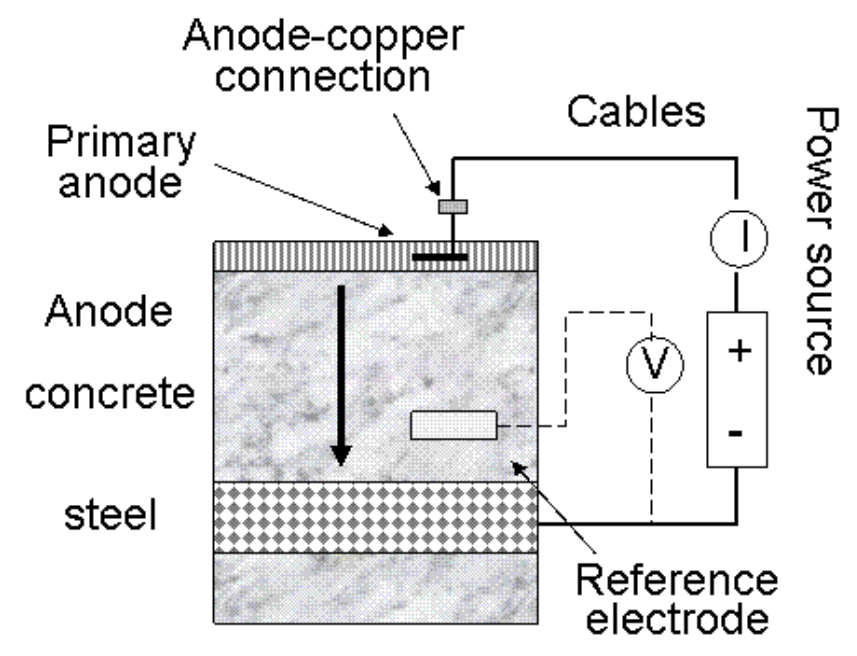

Figure 1-Basic setup of a concrete CP system.

Further components are reference electrodes that operate as monitoring sensors for checking protection and a low voltage power source. In order to ensure that the CP system works properly routine monitoring is performed by electrical measurements (depolarisation) at least twice a year; visual inspection is carried out once a year [9]. Such monitoring is part of a maintenance contract between the CP company and the owner of the structure, that usually runs for ten years and that includes routine checks and replacement of failing components.

The anode system is the most expensive part of a CP system: it may cost between 60 and $90 \%$ of the total cost, depending on the size of the system. Within the anode system, the anode itself (conductive coating, titanium mesh and overlay) is the most expensive part. Primary anodes are the next expensive parts; primary anode-copper connections are third.

\section{FAILURE MODES}

Failure of a CP system (e.g. current stops flowing) causes loss of protection to a degree that depends on the type of component that fails. Various failure modes occur: power units may stop working, anode-copper connections may corrode, reference electrodes may fail and anode materials or primary anodes may degrade. The effect of failure of a component will differ: when a part of the central power system fails, current will stop flowing and the whole system is unprotected. However, if only a part of the system fails this will result in local unprotected areas. Failures in the total CP system will be detected during the next (routine) protection check, within half a year or less. Local failures might be unnoticed during a protection check, but these will be picked up during the yearly visual inspection. Previous work has shown principles, conceptual models for degradation, strategies to avoid such failures and practical observations [10]. The most common types of failure and their effect will be explained below.

Power unit failure will occur instantly (e.g. due to lightning strike) and will cause the whole system to shut down. Primary anode or anode-copper connection failure prevents current flowing to at least part of the system. Reference electrode failure hinders protection checks. Anode 
degradation (due to oxidation of carbon particles or acid formation in the bond plane) increases cell resistance and may cause bond loss. This in turn will cause the need to increase the driving voltage, further accelerating degradation. After some time it becomes impossible to maintain sufficient current for full protection [11]. Global anode failure means the need for complete replacement of the anode, involving high costs. Medium levels of costs are involved for primary anode failure. The other types of failure can be corrected for less money, like replacing a failed power unit.

However, it should be noted that anode system failure does not cause immediate loss of protection. Some of about 700 conductive coating CP systems in a UK motorway complex have shown significant coating degradation (estimated from a photo up to $50 \%$ of the surface), but according to polarization testing, these $\mathrm{CP}$ systems were still working properly, i.e. providing corrosion protection [8]. Similar cases of coating anode degradation but sufficient protection for several years have been observed in the survey reported here. Apparently, there is no simple and hard criterion for degradation related anode system failure. What looks like system failure does not bring about reactivation of corrosion in the short term. Instead, the need for "non-negligible maintenance" is used here as the criterion for working life.

\section{STATISTICAL ANALYSIS OF FIELD DATA}

The statistical technique of survival analysis was used for the analysis of service life of concrete $\mathrm{CP}$ systems and components. In general, survival analysis involves modelling time-to-event data. That is, the data consists of objects (e.g. patients, engines, systems) together with a time until a particular event occurs (e.g. death, failure). See [12] for a comprehensive survey of survival analysis. In this case the events are particular types of failure of CP systems, like global or local failure of the anode or failure of primary anodes, connections or reference electrodes. The goal of the analyses is to quantify and then predict time until failure. One of the difficulties in analysing failure time data is that for some existing objects the time to failure is not known. Reasons can be:

- the object is still working now, or

- after a last known point in time in the past at which the object was still working, there has been no further information on the object, for instance due to lack of monitoring.

This lack of information after a certain point in time is called (right-) censoring. Now, for every object in the group under analysis we can specify a time after which the object has either failed or been censored. There are various techniques that take this censoring into account. One of the results one would like to obtain from the analysis is the survival function $S(t)$, a function in time describing the probability to survive up to and including time $t$, or its complement, the failure function $F(t)=1-S(t)$, indicating the probability to have failed at or before time $t$. In order to estimate the survival function, one can use non-parametric or parametric methods.

The Kaplan-Meier estimator (see [12, Section 1.4]) is called non-parametric because it does not assume a particular distribution type of the survival function (like normal, exponential, etc.). This gives an estimated survival function $S(t)$ or estimated failure function $F(t)=1-S(t)$. The variance can be approximated using the so-called Greenwood's formula. Based on this, approximate $95 \%$ confidence intervals of the failure function can be calculated, i.e. bounds on the estimated $F(t)$, such that the real failure function is within these bounds with $95 \%$ probability. However one must realise that with only a few events of failure, the uncertainty of the estimated failure function will be quite high. 
Using a parametric method, the failure function can be estimated by fitting the censored data to a particular statistical distribution. Here a two-parametric Weibull distribution was used (see [12, Section 2.2.2]), which is one of the most widely used lifetime distributions in reliability engineering. It is a versatile distribution that can take on the characteristics of other types of distributions, based on the value of the shape parameter. Such a parametric method can be useful for obtaining predictions of failure probabilities in the future.

\section{FIELD DATA ON CP SYSTEMS IN THE NETHERLANDS}

In 2011 an inventory was carried out amongst companies in The Netherlands that had installed $\mathrm{CP}$ to concrete structures, as an update to previous surveys performed in 2006 and 2009 [10]. The inventory deals with the following aspects of a CP system: object identification, year of installation, type of structure, protected area and anode type; and required or performed maintenance, repairs, failure and replacement of components. It produced the following observations.

Between 1987 and 2010, about 150 CP systems were installed on various types of concrete structures like bridges, apartment and office buildings, industrial and parking structures with a total protected area of $82,500 \mathrm{~m}^{2}$ of concrete surface. The number of systems per year is shown in Figure 2: about 105 concerned buildings and circa 45 were bridges. In approximately 65 cases conductive coatings were used for the anode compared to around 40 cases in which titanium anodes were used. This is without counting multiple anode types in different parts of a structure (which occurred in 6 cases). Galvanic anodes have been increasingly applied over the last twelve years, to a total of about 35 structures.

From 105 cases sufficient documentation was received for survival analysis. For 45 systems no or insufficient information was available (apart from having been installed), which in most cases means that they are not checked or maintained. For an increasing number of (older) CP systems, recent information is lacking because their maintenance contract was not renewed after 10 years. Because many galvanic systems are not checked on a regular basis, no systematic performance analysis of galvanic CP is possible. Most of the 105 documented cases have been monitored regularly for a longer period of time and have worked well or are still working well. No corrosion related concrete damage was reported. For the discussion of performance, the assumption is made that each CP system was designed, installed and activated properly.

Up until 2010, there were $56 \mathrm{CP}$ systems dating to 2001 or before, so at least ten years old. In three cases, the concrete structure had been demolished (due to redundancy), five were "unknown", which basically means they have been neglected. Twelve had been working well but were no longer monitored. Twenty eight required minor or major ("non-negligible") maintenance. Nineteen systems were still operating well, with only routine maintenance (regular checking). 


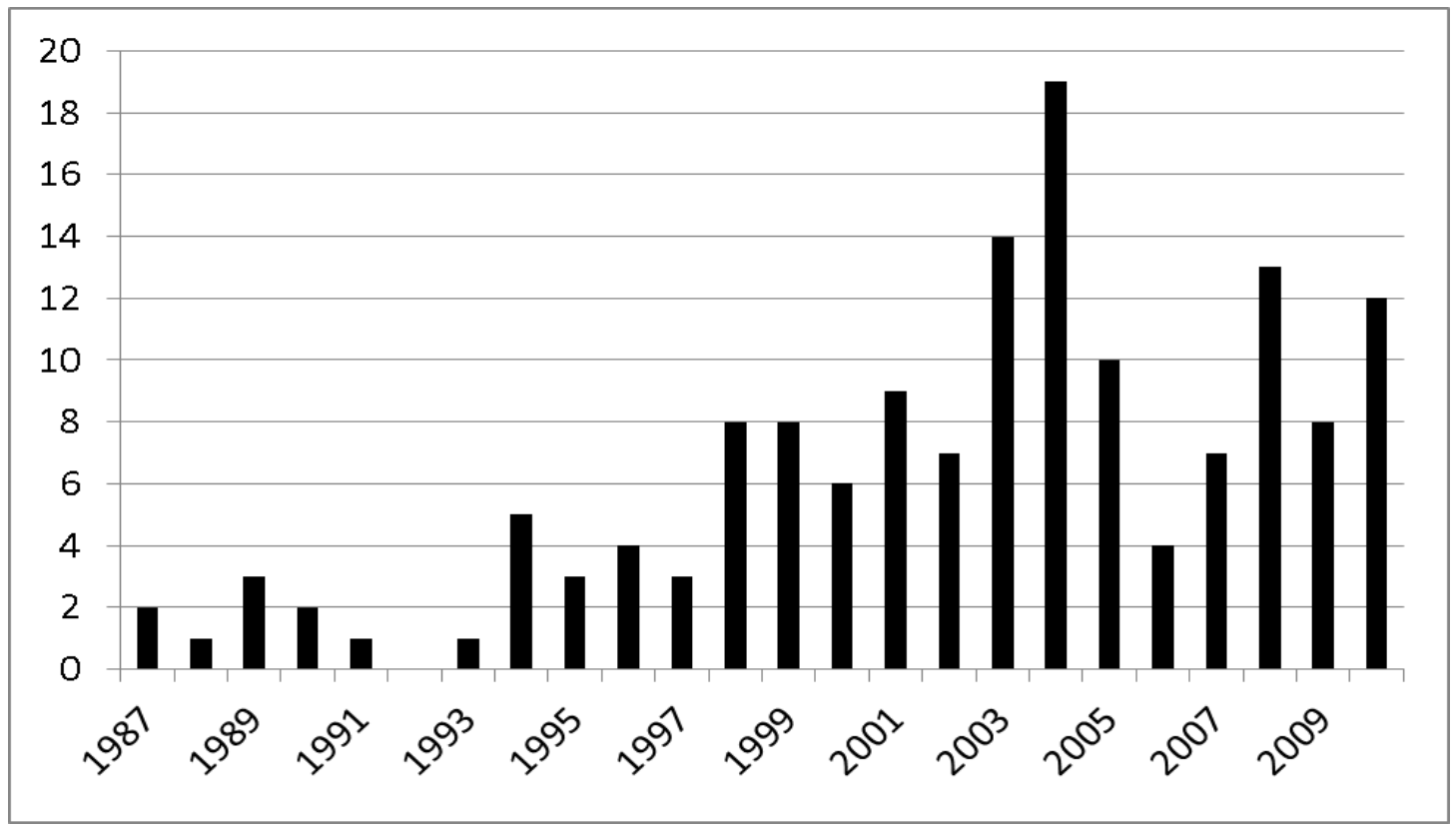

Figure 2 - Number of structures with CP in The Netherlands versus year of installation.

In sixteen cases the anode system failed to some extent, reported as:

- Local poor overall condition of the conductive coating, in seven cases at ages between 3 and 10 years; this was mainly due to local water leakage from overlying parts; the need to replace the anode was signalled in two of these cases, at 7 or 10 years of age; however, anode replacement was not yet carried out and corrosion protection did not seem to be compromised (based on depolarisation testing)

- The anode was replaced, in one case at 7 (a coating) and in one case at 17 years age (a nonmesh titanium type)

- Failure of primary anodes, in seven cases at ages between 5 and 16 years, which were disbonding or all replaced.

In five cases, anode-copper connections were failing and had to be replaced. This occurred at ages between five (one case) and 15 years (two identical cases). In eight cases, power units failed and had to be replaced at ages between 3 and 12 years. In five cases, reference electrodes had to be replaced at ages from 10 years on. A special case is the oldest CP project in the Netherlands. It was installed in 1987, on a bicycle lane of a bridge. Reference electrodes, connections and power units were failing and had to be replaced. These items were repaired for a modest amount of money in 1999 [13]. Since then, it worked properly for at least several years. A few cases were reported suffering from repeated vandalism; others required repairs due to being hit by vehicles. These cases are not further considered as they are non-typical for CP.

The total number of systems younger than ten years was circa 95. Out of these, 55 were monitored and most of them showed good performance without interventions. Minor interventions concerned failing connections (1), primary anode (1), reference electrodes (2), power units (2) and 5 cases of local anode degradation. Around $40 \mathrm{CP}$ systems were not monitored, approx. 35 of these are galvanic systems. 
Summarising the inventory, in about 65 out of 105 documented cases, the CP system worked well without failures or unforeseen maintenance. Of about 56 systems of ten or more years of age, about 19 (33\%) operated without significant interventions. Some 28 older systems required some form of non-negligible maintenance. For a relatively high amount of 17 older and 42 newer systems the present performance was unknown, that is, they were not regularly checked anymore or they had never been checked regularly.

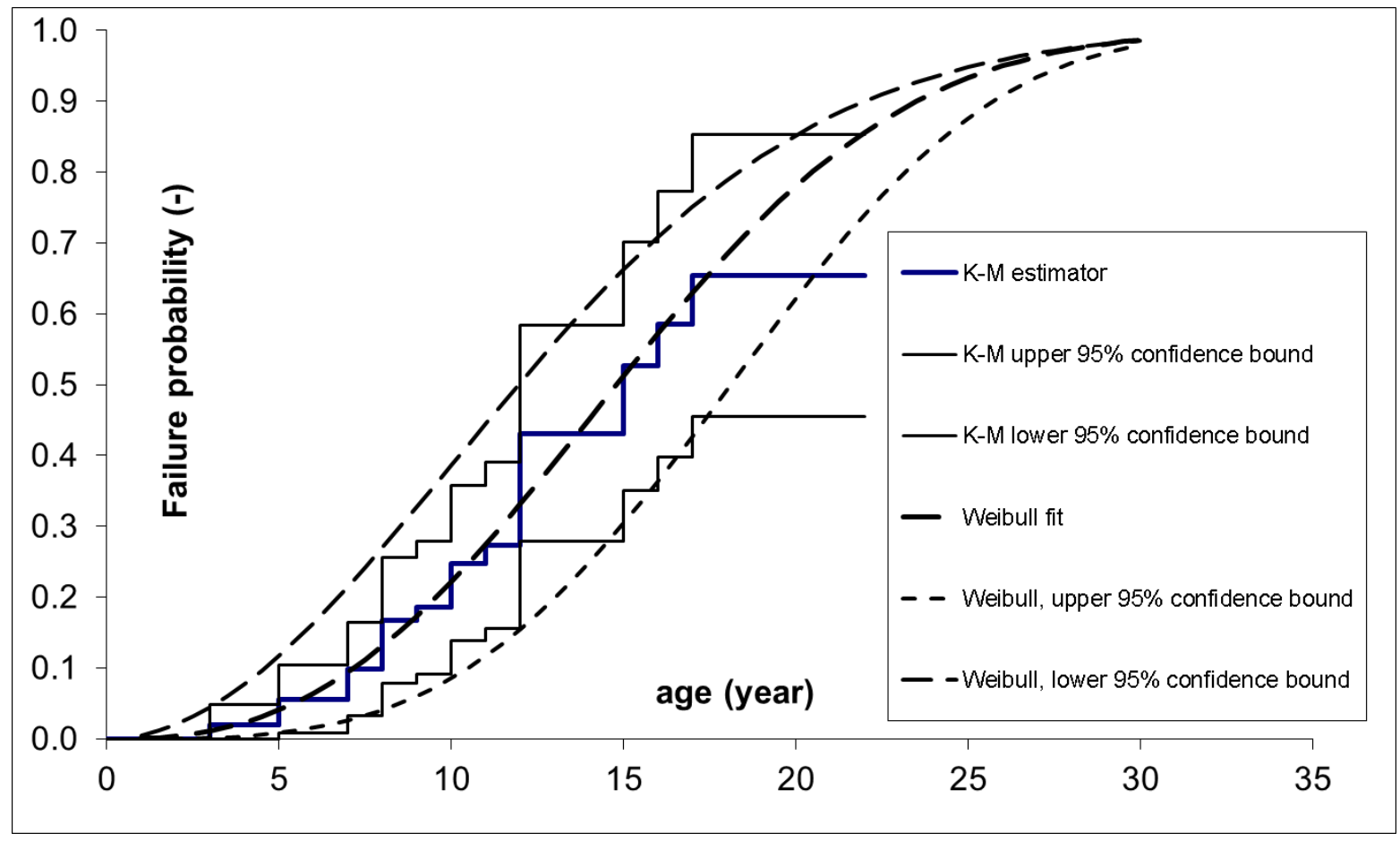

Figure 3 - Failure probabilities for requiring (non-negligible) maintenance of CP system components as a function of age.

\section{DATA ANALYSIS AND DISCUSSION}

This section is aimed at analysing the field data for age-dependent failure of CP system parts. Conductive coatings, primary anodes to them and connections (for both coating and titanium systems) have failed over the years in a number of $\mathrm{CP}$ systems. Occurrence and correcting these types of failure are called non-negligible maintenance in this discussion.

The survival analysis carried out as described above relates the number of failures to the number of CP systems of a particular age; the results are shown in Figure 3. Two types of analysis were used. The solid lines are results of Kaplan-Meier estimates, with the central step-line being the best estimate; upper and lower bounds for $95 \%$ confidence are indicated. The smooth hatched lines were produced by fitting a Weibull distribution to the data, again with the central curve as the best fit with upper and lower $95 \%$ bounds.

Summarising, the graph shows an increasing probability of the need to carry out maintenance with increasing age of CP systems. The two types of analysis produce similar results. It appears from this analysis that there is a $10 \%$ probability that a $\mathrm{CP}$ systems needs maintenance at an age of about 7 years or less, with $95 \%$ confidence this probability lies between 3 to $17 \%$. Moreover, 
there is a $50 \%$ probability that maintenance is needed at an age of 15 years or less; this probability lies between 35 and $70 \%$ with $95 \%$ confidence.

It should be stressed that if a CP system meets this "failure criterion", it does not mean the end of its working life. Actually, all of the systems that needed some maintenance, were still working or were working again after maintenance. Maintenance costs were limited in most cases. Only in two cases did the complete anode system need replacing, costing money of the same order as a new system. For example, simple power units are relatively inexpensive today and anticipating replacement after 10 to 15 years may be a good strategy. Reference electrodes have been improved and/or become cheaper and placing redundant numbers may be a good strategy.

In addition, it should be noted that after some time, steel passivation occurs and current demand decreases. In [8] it is suggested that such a new (second generation) CP system can be designed for lower current density and thus in general can be installed at lower cost.

In the previous section, older and newer CP systems (installed before and after 2001) have been distinguished; possible failure differences between these two groups will be analysed. It should be noted that populations were of similar size: 51 documented and monitored systems were installed up until the end of 2001 (so are aged ten years or more). 55 systems were installed after 2001, aging less than ten years. In a previous study it was postulated that some failures that occurred on early systems, were not seen on newer systems, as if companies had improved critical details [10]. Early failures would represent growing pains of a new industry. This seems to be supported by the present data with regard to connections, power units and possibly for primary anodes. Five cases of connection failure were reported of systems installed until 2001; only one for a system later than 2001. Similarly, power units of older dates failed more frequently in older systems: eight from 2001 or before and only one from the period after 2001. Also older primary anodes failed more frequently than newer ones. In particular with primary anodes, it appears that over time, companies have been experimenting with materials and execution details and seem to have improved them. On the other hand, local anode failure was observed more frequently on newer systems. A clear explanation is not at hand; it may have to do with the fact that in the last ten years more bridges have been equipped with CP (as opposed to more buildings before 2001); which might constitute more aggressive exposure conditions.

\section{LIFE CYCLE COST CALCULATION}

Based on the survival analyses, failure probabilities as a function of time were estimated for the following failure modes: global anode failure; local anode failure; primary anodes; connections; reference electrodes; and power units. The probability curve for primary anode failure for conductive coating anodes is shown in Figure 4. Other failure modes have distinctly different failure rates. Combining these failure probabilities with unit replacement costs for each item, it is possible to predict the expected cost of maintaining the $\mathrm{CP}$ system over a particular period of time. For this purpose, a simple tool is under development (at the moment of writing this paper). The tool is intended to allow companies to better estimate the price of their maintenance contracts and owners to predict the cost of maintaining their structures. Replacement cost levels were estimated for all of these failure types. For example, the cost of replacement of primary anodes was estimated at $€ 20 / \mathrm{m}^{2}$. It should be noted that estimated maintenance cost levels are still subject to comments from participating companies. Other cost categories needed are: initial cost of installing the system and annual costs for inspection and checking and the annual rate of 
interest. Using the data, the life cycle costs for a CP system can be predicted, for example expressed in present value.

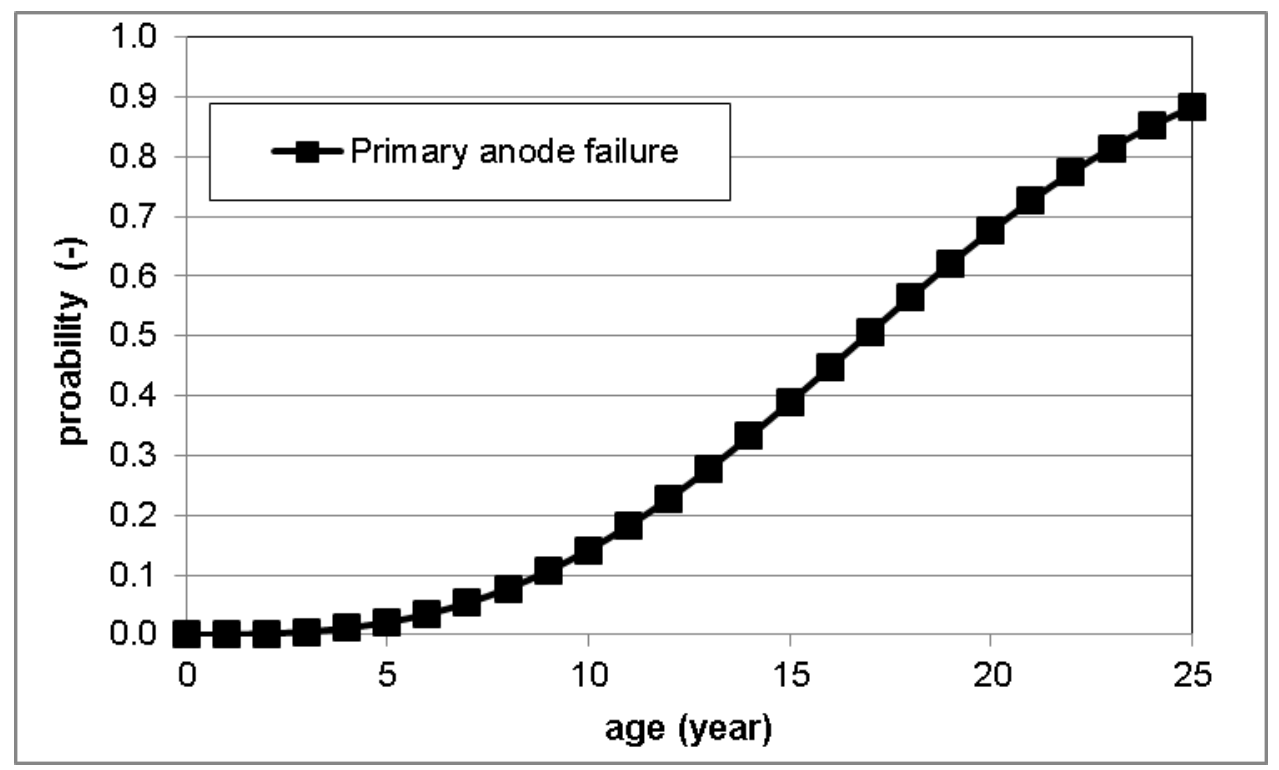

Figure 4 - Probability of failure of primary anodes in conductive coating systems as a function of age.

To demonstrate the calculation, an example case was chosen: a building with $500 \mathrm{~m}^{2}$ of concrete surface, protected using a conductive coating anode and checked manually. The annual rate of interest was set at $2 \%$. The initial cost of installing the CP system was estimated at $€ 75,000$; annual inspection and checkup cost is $€ 2,000$. The development of total cost (including initial installation cost), inspection and maintenance cost and maintenance costs alone (without inspection cost) over 25 years is shown in Figure 5. It can be seen that the actual cost of replacing parts is a relatively small fraction of the maintenance cost. This fraction, however, increases with time. It should be noted that these cost levels are based on expected values and based on the average failure rates for individual items. Using the results from the survival analysis (Figure 3), it is possible to calculate more optimistic or pessimistic values, based on 5\% or $95 \%$ boundaries, respectively. Using those, owners or companies may choose to take a higher risk (of having to spend more money on maintenance in the future) or to stay on the safe side. 


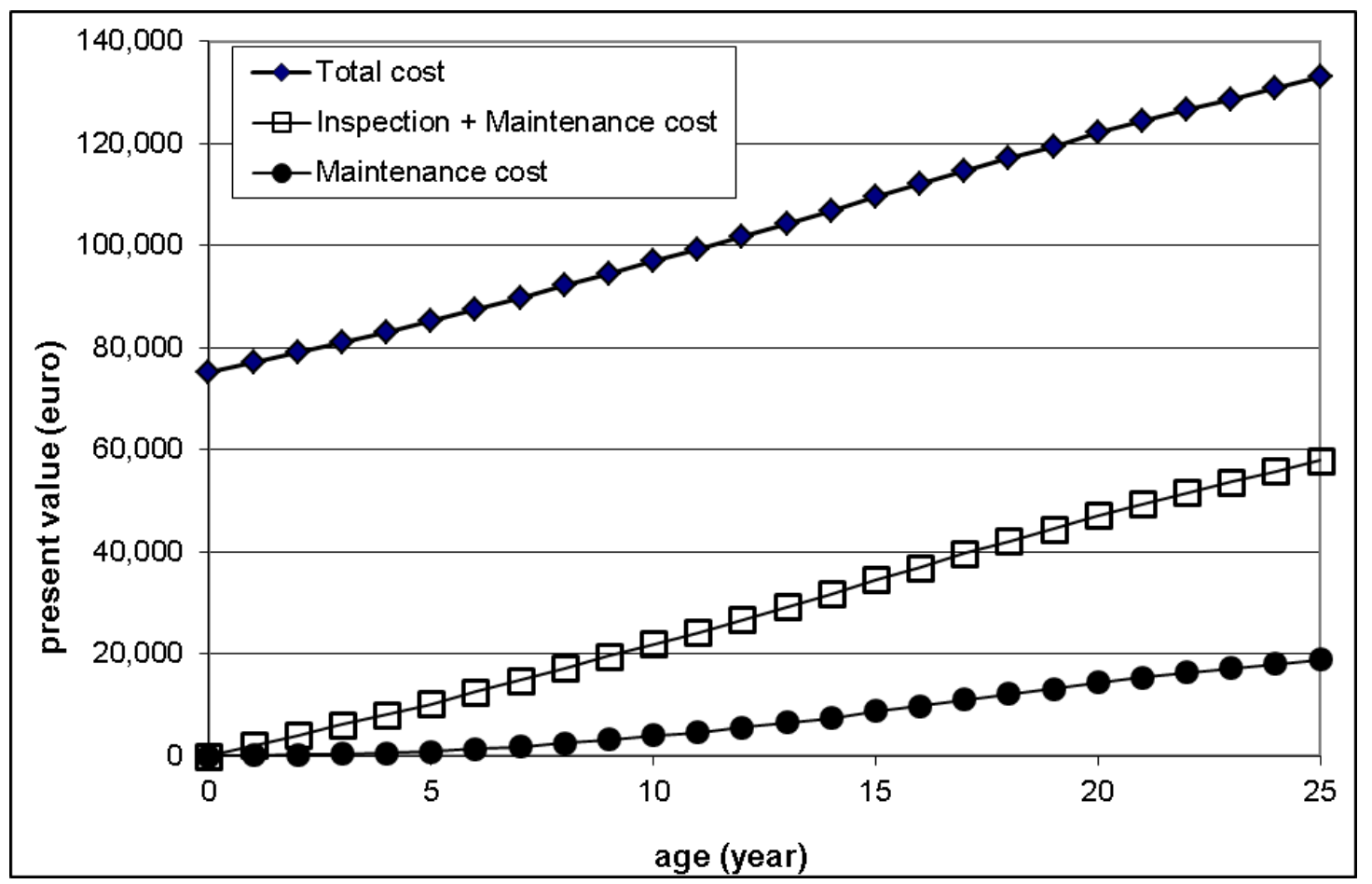

Figure 5 - Development of total cost, inspection plus maintenance cost and maintenance cost alone over time for an example CP system, a building of $500 \mathrm{~m}^{2}$ surface.

\section{CONCLUSIONS}

In order to assess the performance of Cathodic Protection systems in practice, information on 150 concrete structures with CP installed was sought for in an inventory among specialised CP and general concrete repair companies in the Netherlands. Statistical techniques from survival analysis were used for analysing the results. Information on performance and maintenance suitable for such analysis was obtained for 105 cases. Out of these, about 50 had been operating for ten years or longer. About two thirds of the older systems required minor interventions over their existence, that is, replacement of components. Survival analysis was applied to generalise the results, allowing a prediction of the cost of replacing failing components over time. It should be noted that the conclusions below apply only when proper maintenance is carried out, which involves electrical testing for depolarisation at least twice a year and visual inspection once a year, as prescribed by the European CP standard [9] and usually as part of a maintenance contract between the owner and the CP contractor.

The main conclusions from this study are as follows.

Working lives of CP systems without major intervention of ten to twenty years have occurred in practice; corrosion and related damage to concrete has been absent in all documented cases. When intervention was necessary, it was mainly related to defective details such as local leakage and poor electrical isolation. 
Survival analysis of 105 documented cases suggests that (minor) interventions are increasingly necessary with increasing age. It appears that there is a $10 \%$ probability that a $\mathrm{CP}$ system needs maintenance at an age of about 7 years or less. With $95 \%$ confidence this probability lies between 3 to $17 \%$. Moreover, there is a $50 \%$ probability that maintenance is needed at an age of 15 years or less; with $95 \%$ confidence this probability lies between 35 and $70 \%$.

Complete replacement of the anode was carried out in only two cases, one conductive coating anode (on a bridge) and one titanium anode (non-mesh type in a building).

Conductive coatings have shown deterioration in limited numbers, mainly related to local water leakage, which caused the need for local repairs of the coating (ten cases); however, corrosion protection may be provided even if their condition is (visually) poor.

Anodes based on activated titanium have shown long working lives.

Replacement of primary anodes and anode-copper connections was necessary in a number of cases, in particular with older systems; possibly critical details have been improved and the service life of these components has gone up.

Power units and reference electrodes have been replaced in some cases. Simple power units and reference electrodes are relatively inexpensive today, so the cost of these actions is limited.

For an example case it has been shown that life cycle costs of CP systems can be predicted, taking into account failure rates based on the field data. The cost of replacement of components is relatively small compared to the usual cost of inspection and electrical checkups. A simple tool for estimating the cost of maintaining a concrete $\mathrm{CP}$ system is under development.

\section{ACKNOWLEDGMENTS}

The authors gratefully acknowledge the $\mathrm{CP}$ and repair companies who provided information for the inventory: Care4concrete.nl, CORRPRE, ECORemain, RENDON, Van der Heide and Vogel. The work has been made possible by a grant from the Ministry of Economic Affairs under the TNO-co arrangement, project KB Plus Prestatie Toolbox (CP+ Performance Toolbox).

\section{REFERENCES}

1. Bertolini L., Elsener, B., Pedeferri, P. \& Polder, R.B. 2004. Corrosion of Steel in Concrete: Prevention, Diagnosis, Repair. Wiley, Weinheim, ISBN 3-527-30800-8.

2. Tilly, G.P., \& Jacobs, J., 2007. Concrete repairs - performance in service and current practice, IHS BRE Press, Bracknell.

3. Grefstad, K., 2005. Cathodic protection applied on Norwegian concrete bridges. Experience and recommendations. Eurocorr05, Lisboa. (CD-ROM).

4. Nerland, O.C.N, Eri, J., Grefstad, K.A. \& Vennesland, Ø. 2007. 18 years of Cathodic Protection of Reinforced Concrete Structures in Norway - facts and figures from 162 installations. Eurocorr07, Freiburg (CD-ROM).

5. Polder, R.B. 1998. Cathodic protection of reinforced concrete structures in The Netherlands - experience and developments, HERON 43 (1) 3-14. 
6. Wenk, F. \& Oberhänsli, D. 2007. Long-term experience with cathodic protection of reinforced concrete structures, Eurocorr07, Freiburg. (CD-ROM).

7. Tinnea, J.S. \& Cryer, C.B. 2008. Corrosion control of Pacific coast reinforced concrete structures: A Summary of 25 years experience. In: 1st International Conference on Heritage and Construction in Coastal and Marine Environment MEDACHS08, Portugal (CD-ROM).

8. Christodoulou, C., Glass, G., Webb, J., Austin, S. \& Goodier, C. 2010. Assessing the long term benefits of impressed current cathodic protection. Corrosion Science 52, 26712697.

9. CEN, 2000. Cathodic protection of steel in concrete. EN 12696.

10. Polder, R.B., \& Peelen, W.H.A. 2011. Service life aspects of cathodic protection of concrete structures. In Concrete Repair, A practical Guide, M. Grantham (ed.), Taylor and Francis, Abingdon.

11. Mietz, J., Fischer, J. \& Isecke, B. 2001. Cathodic protection of steel-reinforced concrete structures - results from 15 years' experience. Materials Performance 40 (12) 22-26.

12. Kalbfleisch, J.D. \& Prentice, R.L. 2002. The statistical analysis of failure time data. Second Edition, John Wiley \& Sons, New Jersey.

13. Schuten, G., Leggedoor, J., Polder, R.B. \& Peelen, W. 2007. Cost aspects of cathodic protection; a review after 14 years of operation. In Corrosion of reinforcement in concrete, mechanisms, monitoring, inhibitors and rehabilitation techniques, European Federation of Corrosion Publication 38, M. Raupach, B. Elsener, R. Polder, J. Mietz (eds.), Woodhead Publishing Limited, Cambridge, 300-306. 